Rev. Téc. Ing. Univ. Zulia. Vol. 44, No. 1, Enero-Abril, 2021, 29-35

\title{
Bloques de concreto con sustitución de residuos sólidos de polietileno de alta densidad
}

\author{
Derling Jose Mendoza Velazco ${ }^{*}(\mathbb{D})$, Maribel Cecilia Pérez Pirela ${ }^{2}(\mathbb{D}$, Miguel Eduardo \\ Rodríguez Rodríguez ${ }^{3}$ (D), Santiago Andrés Ortiz Montero ${ }^{4}$ (D)
}

${ }^{1}$ Facultad de Ciencias Experimentales, Universidad Nacional de Educacion UNAE, Azogues, C.P. 030154, Ecuador.

${ }^{2}$ Facultad de Ingeniería, Universidad Nacional de Chimborazo UNACH, Riobamba, C.P. 060150, Ecuador. ${ }^{3}$ Facultad de Ciencias Humanísticas y Tecnológicas, Universidad Nacional de Chimborazo UNACH, Riobamba, C.P. 060150 , Ecuador.

${ }^{4}$ Consultoría y Construcción 0 \& B, Investigador independiente, Ambato, C.P. 180103, Ecuador.

*Autor de Correspondencia: derling969@gmail.com

https://doi.org/10.22209/rt.v44n1a04

Recepción: 23 de junio de 2020 | Aceptación: 06 de octubre de 2020 | Publicación: 01 de enero de 2021

\section{Resumen}

\begin{abstract}
El presente estudio tuvo como objetivo analizar la adición de plástico como componente parcial del agregado grueso en el concreto, para el diseño de bloques ecológicos, estableciendo una ecuación lineal que estima la resistencia del bloque a la compresión, variando los volúmenes de cemento, arena, grava y plástico. Para el diseño de los bloques se consideró el volumen en tres proporciones (cemento/agregado fino/agregado grueso), de esta forma se realizaron cuatro tipos de proporciones de mezclas $(1 / 1 / 1 ; 1 / 1,25 / 2,5 ; 1 / 1,5 / 3$ y $1 / 2 / 4)$. El agregado grueso de piedra como componente parcial, fue reemplazado por plástico de tipo polietileno de alta densidad (PEAD) en escamas, en proporciones de 0 ; $25 \mathrm{y}$ $50 \%$ de sustitución pétreo grueso. La resistencia a la compresión - peso unitario del concreto, se midió después de 30 días de curado. La metodología de investigación fue de tipo cuantitativo, de diseño descriptivo experimental. Los resultados fueron analizados mediante un análisis de regresión polinomica lineal en los parámetros, aplicando el paquete estadístico SPSS 25, y su precisión fue juzgada por parámetros nacionales e internacionales, INEN Ecuador 0858 y ASTM C39. Los resultados revelaron que las adiciones de 25 y 50\% de plástico como agregado grueso, reducen el peso unitario del concreto hasta 9,7 y 12,02\%, respectivamente. Pero en tal caso, la reducción de la resistencia a la compresión fue de hasta el 29,17 y $48,5 \%$, respectivamente, para su mejor efectividad, disminuyendo solo el peso unitario de las piezas.
\end{abstract}

Palabras clave: PEAD; plástico; compresión; concreto; bloque; ecuación lineal.

\section{Concrete masonry blocks withscrap HDPE as aggregate}

\begin{abstract}
The present work aims to study the addition of plastic as a partial component of the coarse aggregate in concrete for the design of ecological concrete blocks and to establish a linear equation, to estimate compressive strength for those block, varying cement, sand, gravel, and plastic volume. For the design of blocks, volume in three proportions were considered (cement / fine aggregate / coarse aggregate), this way four types of mix proportions were made (1/1/1,1/1.25/2.5, 1/1.5/3 and 1/2/4). Coarse stone aggregate as a partial aggregate component was replaced by flake HDPE type plastic in 0,25 and $50 \%$ replacement to coarse stone ratios. The compressive strength - unit weight of the concrete was measured after 30 days of curing. The research methodology was of a quantitative type of descriptive experimental design. The results were processed through a linear polynomial regression in the parameters analysis routine, using a statistical software SPSS 25 and their accuracy was assessed by INEN Ecuador 0858 and ASTM C39 parameters. The results revealed that 25 and $50 \%$ additions of plastic as a coarse aggregate reduced the unit weight of concrete to 9.7 and $12.02 \%$ respectively. But in such cases, the reduction of compressive strength was up to 29.17 and $48.5 \%$ respectively for effectiveness improvement reducing only the sample unit weight.
\end{abstract}

Keywords: HDPE; plastic; compression; concrete; brick; linear equation. 


\section{Introducción}

El plástico es un material muy común en nuestra vida diaria. El uso del plástico ha aumentado sustancialmente en la última década, ya que es ligero, resistente a la humedad y a la corrosión, duradero y relativamente de bajo presupuesto [1]. El plástico ha sustituido a muchos materiales tradicionales como madera, piedra, hueso, cuero, papel, metal, vidrio y cerámica. Hoy en día, casi todos los materiales que utiliza el ser humano están diseñados de plástico. Como resultado, la producción de plástico aumentó exponencialmente de 1964 a 2020 [2]. La producción de plásticos ha aumentado de 15 a 311 millones de toneladas métricas. Si esta tendencia continúa, se espera que la producción de plástico se duplique en 20 años y casi se cuadruplique para el 2050 [3]. Junto con la producción, la cantidad de residuos plásticos también está aumentando exponencialmente. Debido al insuficiente reciclaje, cada año se generan millones de toneladas de desechos plásticos, que terminan en los vertederos y en los océanos. En los vertederos se eliminan entre el 22 y el $43 \%$ de los plásticos y se vierten al menos 8 millones de toneladas de plásticos en el océano $[4,5]$.

En Ecuador, la primera industria del plástico comenzó en 1986. Desde entonces, el consumo de plástico ha ido aumentando día a día. Solo en la ciudad de Quito, entre el total de desechos sólidos, el plástico era el 4,15\% en 2005 y el 5,46\% en 2014 [6], lo que indica el aumento de la tasa de desechos de plástico. Los desechos plásticos pueden convertirse en un recurso potencial, si se pueden reciclar. Uno de esos intentos es que puedan utilizarse en el concreto, solidificando estos desechos [7].

Se han realizado varios estudios para evaluar la aplicabilidad de diferentes tipos de plásticos con fines de construcción. Jagdish et al [8] evaluaron las propiedades del concreto con plástico reciclado como agregado grueso, en un $5,10,15$ y $20 \%$ de sustitución de la piedra. Utilizaron plástico HDPE y prepararon un total de 90 cilindros y 5 vigas. Luego, a los 7, 14 y 28 días de curado, se analizaron los resultados de la resistencia a la compresión, la resistencia a la tracción dividida, la resistencia a la flexión y la densidad seca de los especímenes. La relación agua/ cemento fue de $0,5 \mathrm{y}$ la proporción de la mezcla fue de $1 / 1,8 / 3$ en base al volumen. De acuerdo con su resultado, la máxima reducción de la resistencia a la compresión fue del $44 \%$ para un $20 \%$ de sustitución de piedra por plástico reciclado. La resistencia a la tracción y a la flexión del concreto se redujo con el aumento del porcentaje de plástico reciclado. La densidad seca se redujo en un 1,5\% por cada $5 \%$ de sustitución de piedra. Concluyeron el estudio señalando que hasta un $15 \%$ de sustitución de agregados de piedra por plástico reciclado, es viable su uso para la aplicación estructural.

Otro trabajo realizado por Subramani y Pugal [9] sobre la sustitución parcial del agregado grueso por el polihidroxibutirato (PHB), que es un plástico biodegradable [10], donde se reemplazó 5, 10 y 15\% del agregado grueso por plástico y la relación agua/cemento fue de 0,46 . Se observó que el $20 \%$ de los agregados de desechos plásticos pueden ser reemplazados sin efectos perjudiciales a largo plazo, y con propiedades de desarrollo de resistencia aceptables. En la experimentación de Akila et al.[11] se demostró una gran aplicabilidad de las bolsas de plástico, como agregado fino en el concreto. El agregado fino de plástico se producía calentando $\left(178^{\circ} \mathrm{C}\right)$ las bolsas de plástico, seguido de un enfriamiento $\left(30{ }^{\circ} \mathrm{C}\right)$; los desechos líquidos luego se enfriaban y eran procesados en una máquina trituradora, que producía escamas de plástico con un módulo de finurade $4,7 \mathrm{~mm}$, después el $10,20,30$ y $40 \%$ de agregado fino fue reemplazado por el agregado fino de plástico. Después de 28 días de curado, se evaluó la capacidad de trabajo, densidad aparente, prueba de velocidad de pulso ultrasónico, resistencia a la compresión y a la flexión de los especímenes. Así, la resistencia aumentó significativamente con la presencia de residuos de bolsas de plástico. Se llegó a la conclusión que la bolsa de plástico puede utilizarse con éxito para sustituir a los agregados finos convencionales en el concreto.

Según Kamaruddin et al. [12] existen efectos positivos al aplicar plástico en el concreto. Para Roni et al. [13] los costos de producción de los bloques de concreto varían de forma considerable según la calidad, resistencia y fines de utilidad de los mismos; el modelo estándar de dimensión 40×20×20 cm tiene un costo de 14 centavos de dólar americano. Para este propósito, los autores citados anteriormente prepararon 12 cubos de dimensión 150x150x150 $\mathrm{mm}^{3}$, con la proporción de agua-cemento variando el porcentaje $(0,10,30$ y $50 \%)$ de la colección de plástico. Utilizando un tamaño máximo de $13 \mathrm{~mm}$ de agregado plástico. Con los resultados se demostró que la densidad aparente de la mezcla se reduce con la ganancia en la sustancia plástica, por lo que la densidad aparente es más baja que en el concreto convencional. De igual forma, la reducción de la densidad aparente fue directamente relacionada con la recolección de plástico. La densidad del material se redujo en un 2,5; 6 y 13\% respectivamente, por lo que la reducción de la densidad se debió al bajo peso unitario del plástico.

En este estudio, la resistencia a la compresión y el peso unitario del concreto, se clasificaron considerando cuatro relaciones de mezcla (basadas en el volumen) con plástico como reemplazo parcial ( 25 y $50 \%$ ) del agregado grueso. Se indagó acerca de las relaciones de mezcla que pueden utilizarse tanto para fines estructurales como no estructurales según [14 y 15]. También se propuso un modelo matemático cuya adecuación se evaluó utilizando diferentes parámetros estadísticos. La clasificación de los bloques de concreto usada en el presente trabajo, se presenta en la Tabla 1 [14-15]. 
Tabla 1. Dimensiones de los bloques de concreto [14-15].

\begin{tabular}{ccccccc}
\hline \multicolumn{3}{c}{ Dimensiones nominales del Instituto Ecuatoriano de } & \multicolumn{3}{c}{ Dimensiones reales } \\
Normalización & Ancho (cm) & Alto (cm) & Largo (cm) & Ancho (cm) & Alto (cm) \\
\hline Tipo & Largo (cm) & Ancho & 20 & 39 & $19,14,09$ & 19 \\
C, B, D & 40 & $20,15,10$ & 20 & 39 & $09,14,19$ & 19 \\
E & 40 & $10,15,20$ & 20 & 39 & $09,14,19,24$ & 20 \\
\hline
\end{tabular}

\section{Materiales y métodos}

Para llevar a cabo el estudio se seleccionaron las materias primas, que fueron, cemento Portland Tipo I ASTM C150, con un 65\% de cemento Chimborazo® que posee $35 \%$ de yeso, con el fin de retardar el tiempo de fraguado; agregado fino con un módulo de finura de 2,7; agregado grueso de $19 \mathrm{~mm}$; residuos de polietileno de alta densidad de tipo termoplástico (PEAD) virgen ( $\sin$ haber sido reprocesado), con un índice de fluidez de $77 \mathrm{~g} / 10 \mathrm{~min}$, absorción de agua mg a $96 \mathrm{~h}<0,5$; densidad estándar de 0,963 g/ $\mathrm{cm}^{3}$, módulo de flexión de 15,396 Kg/ $\mathrm{cm}^{2}$, resistencia a la tensión de ruptura de $155 \mathrm{Kg} / \mathrm{cm}^{2}$, resistencia al impacto izod de $13 \mathrm{Kg} . \mathrm{cm} / \mathrm{cm}$ y elongación de 555\%. De igual forma el PEAD presentaba un módulo de finura de $5,91 \mathrm{~mm}$, peso unitario: $3,5 \mathrm{KN} / \mathrm{m}^{3}$, relación agua/cemento por peso de 0,61 (sin aire incluido).

Para mezclar los materiales, se seleccionaron con base en el volumen cemento/agregado fino/agregado grueso (pétreo triturado y plásticos reciclados), aplicando los siguientes cuatro conjuntos: $1 / 1 / 1 ; 1 / 1,25 / 2,5$; $1 / 1,5 / 3$ y $1 / 2 / 4$. Para cada conjunto, los agregados gruesos fueron reemplazados por $0 ; 25$ y $50 \%$ de plástico, medidas porcentuales estándar recomendadas por Olonade et al. [16] para el diseño de bloques de concreto con agregados de plástico.

Para preparar las muestras se utilizó un molde cilíndrico limpio y seco de $1000 \mathrm{~mm}$ de diámetro y 1000 $\mathrm{mm}$ de altura. Se diseñaron tres muestras para cada reemplazo. El concreto fue mezclado de forma manual. El material moldeado fue ubicado en bloques de dimensión rectangular $40 \times 20 \times 20 \mathrm{~cm}^{3}$, luego fue reposado por un lapso de 24 horas a temperatura ambiente. Los bloques o prototipos se sacaron del molde rectangular con cuidado. Justo después de sacarlos del molde, los materiales se sumergieron completamente en un tanque de curado durante 30 días; método recomendado por Akinyele y Ajede[17] al aplicar el tanque de curado UTC-0960 de metal con una temperatura media de control entre 38 y 40 ${ }^{\circ} \mathrm{C}$ con $\pm 2^{\circ} \mathrm{C}$ de precisión. Según Shirish [18] el curado de bloques de concreto con materiales anexos como plástico, cal, yeso, cenizas, entre otros, aplicando un tanque para curado grande con dimensiones de 800x1800x950 mm, puede desarrollarse con un tiempo prolongado entre 28 y 30 días, los cuales fueron usados durante el presente estudio.

Todo el procedimiento de preparación de las muestras se llevó a cabo siguiendo las normas internacionales de cemento y concreto de la Sociedad Americana para Pruebas y Materiales (ASTM, según sus siglas en inglés), donde el ensayo normalizado para resistencia a la compresión de especímenes de bloques de concreto, corresponde a los códigos ASTM C39, C31, C150, C617, C1077 y C1231[19]. Después de 30 días de curado, los bloques fueron retirados del tanque de curado. Seguidamente fueron medidos en peso y se les realizó el ensayo de resistencia a la compresión. Los resultados fueron comparados con los estándares de NTE INEN [1928].

\section{Resultados y discusión}

En esta sección se analizan los resultados de la adición de plástico como componente parcial del agregado grueso en el concreto, para el diseño de bloques ecológicos. Después de 30 días de curado se observó que, tanto la resistencia a la compresión como el peso unitario ,se redujeron a medida que aumentaba el porcentaje de plástico para las cuatro relaciones de mezcla (Figura 1). Con base en estos resultados, y de acuerdo a lo señalado por Shirish [18] y las normas técnicas NTE INEN [20-21], el propósito de uso de las muestras se incluye en la Figura 2.

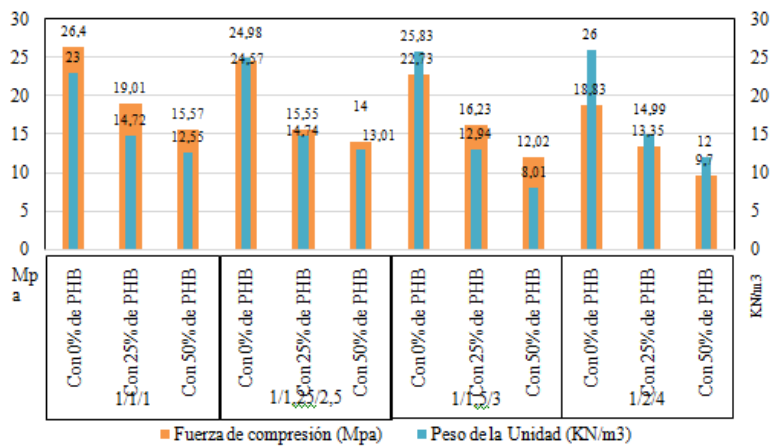

Figura 1. Resistencia a la compresión y variación del peso unitario con el aumento del porcentaje de plástico para cada proporción. 


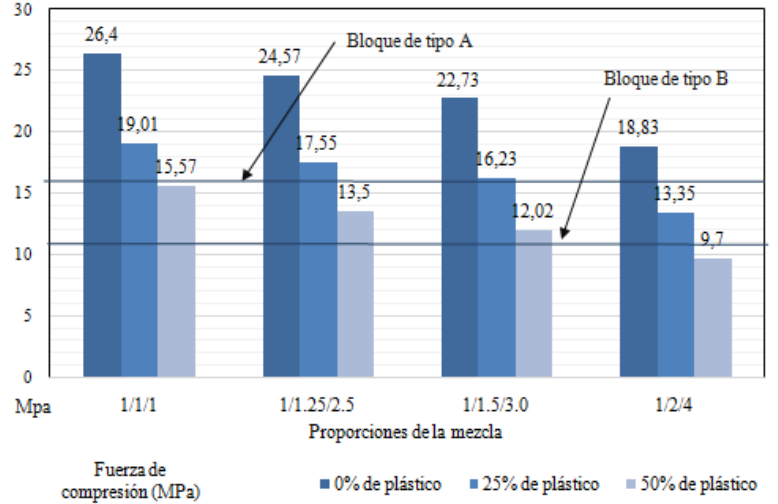

Figura 2. Resultados de la fuerza de compresión aplicados a los bloques de concreto diseñados con plástico como componente parcial del agregado grueso de concreto.

En la Figura 2 se graficaronn los resultados de las fuerzas de compresión que se obtuvieron con los bloquescreados, al reemplazar con $25 \%$ de plástico el agregado grueso de piedra con una proporción de 1/1/1. De igual manera, las muestras de concreto con $25 \%$ de plástico con proporción de 1/1,25/2,5 y las muestras de concreto con un $25 \%$ de plástico a una proporción de $1 / 1,5 / 3$; mostraron una resistencia de un bloque tipo A. Los bloques tipo A según NTE INEN 0638 [29], se usan en paredes exteriores de carga sin revestimiento y poseen una $\mathrm{MPa} \geq 16$. A excepción de la muestra con $25 \%$ de plástico a una proporción de $1 / 2 / 4$, la cual exhibió una MPa de 13,35; estando por debajo del índice de 16 MPa.

Los resultados de las fuerzas de compresión que se obtuvieron con los bloquesde concreto al reemplazar el $50 \%$ de plástico del agregado grueso con una proporción de $1 / 1 / 1,1 / 1,25 / 2,5$ y las muestras de concreto con proporción de 1/1,5/3; demostraron tener la resistencia de un bloque tipo B. Los bloques tipo B, de acuerdo con NTE INEM0862 [30] se usan en paredes exteriores de carga con revestimiento y en paredes interiores de carga con o sin revestimiento. Estos, mostraron un rango de $\mathrm{MPa} 16 \leq \mathrm{MPa} \geq 11$, a excepción de la muestra con $50 \%$ de plástico a una proporción de $1 / 2 / 4$, que presentó una MPa de 9,7 la cual se encuentra por debajo del índice de $11 \mathrm{MPa}$.

\section{Desarrollo empírico de ecuaciones}

A partir de los resultados de la prueba anterior, se dio respuesta a la segunda parte del objetivo objetivo del presente trabajo, mediante la propuesta de una al proponer una ecuación de regresión polinómica lineal en los parámetros (Ecuación 1), utilizando el Software Statistical Package for the Social Sciences (SPSS) versión 25. $f_{c}^{\prime}=-35520,66 \mathrm{~V}_{\mathrm{C}}-98859,50 \mathrm{~V}_{\mathrm{A}}-55353,74 \mathrm{~V}_{\mathrm{Pi}}-71313,92 \mathrm{~V}_{\mathrm{P} 1}+181,29(1)$

En esa ecuación se mantuvo la resistencia a la compresión de 30 días $\left(f_{c}^{\prime}\right.$ en $\mathrm{MPa}$ ) como la función del volumen $\left(\mathrm{en} \mathrm{m}^{3}\right)$ de cemento $\left(\mathrm{V}_{\mathrm{C}}\right)$, arena $\left(\mathrm{V}_{\mathrm{A}}\right)$, piedra $\left(\mathrm{V}_{\mathrm{pi}}\right)$ y plástico $\left(\mathrm{V}_{\mathrm{Pl}}\right)$. Los valores de las variables de entrada se muestran en la Tabla 2 (como se utilizó en el experimento).

Considerando a Ruma y Well[31], el coeficiente de correlación de Pearson puede tomar valores entre -1 y 1. La correlación de una variable con ella misma siempre es igual a 1 . El valor del coeficiente de correlación puede variar de $-1 \mathrm{a}+1$. Para la correlación de Pearson, un valor absoluto de 1 indica una relación lineal perfecta, mientras que una correlación cercana a 0 , indica que no existe relación lineal entre las variables. El valor obtenido para la ecuación fue de 0,97; lo cual indica una correspondencia aceptable en la covariación de tipo lineal.

Como segundo criterio de eficiencia, se aplicó el coeficiente de determinación, de acuerdo con Harel [32], que se define como la proporción de la varianza total de la variable explicada por la regresión. El coeficiente de determinación, denotado como $\mathrm{R}^{2}$, refleja la bondad del ajuste de un modelo a la variable que se pretende explicar. Es importante destacar que el valor de $\mathrm{R}^{2}$ establece un rango entre 0 y 1 . El resultado para la ecuación propuesta fue de 0,94; como un coeficiente cercano de 1 .

Con el tercer criterio de eficiencia, para la validación de la ecuación lineal se desarrolló el error absoluto medio (MAE, según sus siglas en inglés), con un valor de 0,93; el caul permite determinar la medida de las diferencias medias entre los valores pronosticados y observados [33].

El coeficiente de eficiencia (E) y el coeficiente de eficiencia modificado miden principalmente la eficacia, pero también la eficiencia de la ecuación lineal empírica, en la búsqueda del máximo potencial de eficiencia, mediante la reducción o aumento de residuos de plásticos en los componentes agregados gruesos, con un valor de $0,94 \mathrm{y}$ 0,77 ; ambos con un valor oscilante de $1 \geq \mathrm{E}>5$, considera eficiente, según Geethu and Santhoshkumar [34].

Finalmente, entre los criterios de eficiencia se aplicaron el índice del acuerdo (D) y el índice de acuerdo modificado, cuyos valores de 0,99 y 0,88; representando una aportación en la ecuación lineal, básicamente mediante la incorporación de su fórmula de corrección, que excluye la concordancia debida exclusivamente al azar. Esta corrección relacionada las distribuciones marginales de acuerdo con conGeethu and Santhoshkumar [33]. En este sentido, la Ecuación 1 es válida dentro del rango de parámetros aplicables que se muestra en la Tabla 4. 
Tabla 2. Variables de entrada (volumen en $\mathrm{m}^{3}$ ) y fuerza de compresión (en MPa).

\begin{tabular}{|c|c|c|c|c|c|c|c|}
\hline \multirow{2}{*}{$\begin{array}{l}\text { Proporción de } \\
\text { la mezcla }\end{array}$} & \multirow{2}{*}{$\begin{array}{l}\text { Contenido de } \\
\text { plástico }(\%)\end{array}$} & \multirow{2}{*}{$\begin{array}{l}\text { Cemento } \\
\left(\mathrm{m}^{3}\right) \times 10^{-4}\end{array}$} & \multirow{2}{*}{$\begin{array}{c}\text { Arena } \\
\left(\mathrm{m}^{3}\right) \times 10^{-4}\end{array}$} & \multirow{2}{*}{$\begin{array}{c}\text { Piedra } \\
\left(\mathrm{m}^{3}\right) \times 10^{-4}\end{array}$} & \multirow{2}{*}{$\begin{array}{l}\text { Plástico } \\
\left(\mathrm{m}^{3}\right) \times 10^{-4}\end{array}$} & \multicolumn{2}{|c|}{ Fuerza de compresión $(\mathrm{MPa})$} \\
\hline & & & & & & $\begin{array}{c}\text { Datos } \\
\text { experimento }\end{array}$ & $\begin{array}{c}\text { Datos } \\
\text { ecuación }\end{array}$ \\
\hline \multirow{3}{*}{$\Xi$} & 0 PEAD & 8,3 & 8,3 & 8,3 & 0 & 26,40 & 23,81 \\
\hline & 25 PEAD & 8,3 & 8,3 & 6,3 & 2 & 19,01 & 20,62 \\
\hline & 50 PEAD & 8,3 & 8,3 & 4,2 & 4,2 & 15,57 & 16,56 \\
\hline \multirow{3}{*}{ 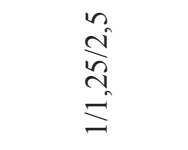 } & 0 PEAD & 5 & 6,7 & 13,3 & 0 & 24,57 & 23,68 \\
\hline & 25 PEAD & 5 & 6,7 & 9,7 & 3,4 & 17,55 & 18,25 \\
\hline & 50 PEAD & 5 & 6,7 & 6,65 & 6,65 & 13,50 & 13,06 \\
\hline \multirow{3}{*}{$\stackrel{m}{\lesssim}$} & 0 PEAD & 4,5 & 6,8 & 13,6 & 0 & 22,73 & 22,80 \\
\hline & 25 PEAD & 4,5 & 6,8 & 10,1 & 3,5 & 16,23 & 17,22 \\
\hline & 50 PEAD & 4,5 & 6,8 & 6,8 & 6,8 & 12,02 & 11,95 \\
\hline \multirow{3}{*}{$\stackrel{+}{\stackrel{\Delta}{\Delta}}$} & 0 PEAD & 3,6 & 7 & 14,4 & 0 & 18,83 & 19,59 \\
\hline & 25 PEAD & 3,6 & 7 & 10,8 & 3,6 & 13,35 & 13,85 \\
\hline & 50 PEAD & 3,6 & 7 & 7,2 & 7,2 & 9,70 & 8,10 \\
\hline
\end{tabular}

Cabe señalar que todos los valores volumétricos de la Tabla 2 corresponden a una muestra de bloque de concreto hueco de $40 \times 20 \times 20 \mathrm{~cm}^{3}$. La precisión de la ecuación de regresión polinómica lineal en los parámetros (Ecuación 1), se muestra en la Tabla 3, pudiéndose decir que la misma se ajusta bien a los valores experimentales.

Tabla 3. Valor de los criterios de eficiencia.

\begin{tabular}{cc}
\hline Criterio de eficiencia & Valores \\
\hline Coeficiente r de Pearson & 0,97 \\
Coeficiente de determinación, $\mathrm{R}^{2}$ & 0,94 \\
Significado de error absoluto medio, MAE & 0,93 \\
Coeficiente de eficiencia, E & 0,94 \\
Coeficiente de eficiencia modificado & 0,77 \\
Índice de acuerdo, D & 0,99 \\
Índice de acuerdo modificado & 0,88 \\
\hline
\end{tabular}

Tabla 4. Rango de parámetros aplicables para la Ecuación 1 (para una muestra de bloque hueco de concreto $40 \times 20 \times 20 \mathrm{~cm}^{3}$ ).

\begin{tabular}{cc}
\hline Parámetro & $\begin{array}{c}\text { Rango de volumen en una muestra } \\
\left(\mathbf{m}^{\mathbf{3}} \mathbf{)} \mathbf{x} \mathbf{1 0}^{-4}\right.\end{array}$ \\
\hline Cemento & $3,6 \leq \mathrm{V}_{\mathrm{C}} \leq 8,3$ \\
Arena & $6,7 \leq \mathrm{V}_{\mathrm{A}} \leq 8,3$ \\
Piedra & $4,2 \leq \mathrm{V}_{\mathrm{pi}} \leq 14,4$ \\
Plástico & $0 \leq \mathrm{V}_{\mathrm{Pl}} \leq 7,2$ \\
\hline
\end{tabular}

Los resultados demuestran que la resistencia a la compresión de los bloques de concreto, que involucran el plástico como sustitución parcial, probablemente sea significativamente menor al ordinario o concreto controlado. Es mucho más claro cuando el porcentaje de contenido de plástico, como sustitución parcial, aumenta cuanto más baja sea la resistencia del concreto. En cuanto a la densidad de la masa para el concreto ordinario y plástico, se demostró que el concreto plástico definitivamente posee un peso menor en comparación al concreto ordinario, ya que está hecho de plástico. Esto muestra que el plástico sería una buena plataforma para la producción de concreto ligero, produciendo una buena resistencia a la compresión.

Aunque investigadores como Edmund et al. [35] logran obtener una satisfactoria resistencia a la compresión, puede que, debido al bajo porcentaje de contenido de plástico utilizado, así como a los tipos de plásticos utilizados, se obtenga un importante valor o coeficiente, ya que algunos materiales plásticos son más duros y fuertes que otros, como el polipropileno. Aparte del bajo porcentaje de plástico utilizado, Edmund et al. [35] recomiendan la adición de solución plastificante, para aumentar o mejorar la mezcla con el plástico, como sustituto del agregado grueso o piedras. Igualmente, se puede recomendar el plástico como sustituto de los áridos finos en el concreto, logrando producir un resultado mucho mejor en comparación con la sustitución del agregado grueso.

\section{Conclusiones}

El presente estudio brinda la oportunidad de contribuir a la reducción de la contaminación ambiental por plásticos, debido al rápido aumento de estos desechos 
en la República del Ecuador. Por lo tanto, el reciclado de los desechos de plástico en el concreto, como agregado grueso, puede ser una solución eficaz para eliminar una gran cantidad de plástico que puede reducir en gran medida la contaminación ambiental y producir un concreto categorizado de forma ambiental, como concreto verde.

La adición de plástico disminuye el peso unitario del concreto, pudiéndose utilizar para producir bloques de concreto ligero, cuya resistencia del concreto sustituido, con 25 y un $50 \%$ de plástico como agregado grueso, resulta suficiente para ser utilizado con fines no estructurales de alta carga como edificios o departamentos de un solo piso o nivel.

Finalmente, se puede mencionar quelos costos de producción de los bloques de concreto con sustitución de residuos sólidos de polietileno de alta densidad, tienen un valor económico menor que los bloques convencionales de concreto, los cuales poseen un costo de 14 centavos de dólar, mientras que, estos bloques ecológicos tienen uno de 11 centavos de dólar cada uno. El valor de producción disminuye 3 centavos de dólar estadounidense, (pero el proceso y acceso del plástico parece como componente parcial del agregado grueso, beneficiando al ambiente al aglutinarse con el cemento).

\section{Referencias bibliográficas}

[1] Chalmin, P.: "La historia de los plásticos: desde el Capitolio hasta la Roca Tarpeya".Field Actions Science Reports, Vol. 19 (2019), 6-11.

[2] Chuanxiu, J., Zhang, M. y Lu, P.: "Preparación y caracterización de la urea recubierta de poliuretano/TM nano compuesto como fertilizantes de liberación controlada". PolymerPlastics Technology and Materials, Vol. 59, No. 9 (2020), 975-984.

[3] 3. Moriet, A., Bleriot J., Opsomer, R., Widmer, S., Banks, I., DeSmet, M., Murphy, J., Struchery, M., Swartz, S. y Vanthournout, H.: "Foro Económico Mundial, La nueva economía de los plásticos Repensando el futuro de los plásticos". Lenaïc Gravis., Barcelona, (2016).

[4] Van Emmerik, T. y Schwarz, A.: "Los desechos plásticos en los ríos". WIREs Water, Vol. e1398, (2020), 1-13.

[5] Gourmelon, G.: "La producción mundial de plástico aumenta”. Recycling Lags, Vital Signs, Vol. 11, (2015), 1-7.

[6] Veletanga, F.: "Estudio de la huella ecológica de plástico PET (Tereftalato de polietileno) del barrio Nueva Aurora en la Parroquia de Guamaní en el distrito metropolitano de Quito". Universidad Central de Ecuador., Ecuador, (2017).
[7] Awoyeraa, P. y Adesinab, A.; "Los residuos plásticos a los productos de construcción: Situación, limitaciones y perspectivas futuras". Case Studies in Construction Materials, Vol. 12, No. e00330, (2020), 1-11.

[8] Jagdish, K., Gohil, G y Patel, N.: "Estudio experimental sobre el uso de residuos plásticos como reemplazo parcial de los agregados gruesos y finos en el hormigón". International Journal for Research in Applied Science \& Engineering Technology (IJRASET), Vol. 6, No. 4 (2018), 35613567.

[9] Subramani, T. y Pugal, V.: "Estudio experimental sobre los residuos plásticos como agregado grueso para el hormigón estructural".International Journal of Application or Innovation in Engineering \& Management, Vol. 4, No. 5 (2015), 2319 - 2347.

[10] Chávez- Romero, S., Aulestia-Viscarra, Sh., Cando, W., Bucheli, J. y Fernández, L.: "Evaluación de las propiedades mecánicas de una mezcla asfáltica en caliente modificada por vía seca con tapas plásticas de polipropileno". Revista Técnica. De la Facultad de Ingenieria, Vol. 42, № 3, (2019), 104151.

[11] Akila, S., Ajithkumar, C., Deepankumar, S., Jeeva, P. y Senthilkumari, T.: "Utilización de desechos plásticos como reemplazo parcial del agregado fino en la mezcla de hormigón". International Journal of Advanced Science and Engineering Research, Vol. 3, No. 1 (2018), 214-219.

[12] Kamaruddin, M., Abdullah, M., Zawawi, M y Zainol, M.: "Uso potencial de los residuos plásticos como materiales de construcción: Progresos recientes y perspectivas futuras". IOP Conference Series: Materials Science and Engineering, Vol. 267, No. 012011, (2017), 1-10.

[13] Roni, S., Stryjer, R., Weizman, A. y Nutt, D.: "Spon's Architects' and Builders' Price Book 2013". Taylor \& Francis Group, Londres, (2013).

[14] Norma Ecuatoriana de la Construcción - NEC: NEC-SE-HM Estructuras de Concreto Armado. Ministerio de Desarrollo Urbano y Vivienda, (2014). https://www.habitatyvivienda.gob.ec/ wp-content/uploads/downloads/2014/08/NECSE-HM.pdf.

[15] Instituto Ecuatoriano de Normalización, INEN, Casilla 17-01-3999 - Quito-Ecuador, (1993). https://www.compraspublicas.gob.ec/ ProcesoContratacion/compras/PC/bajarArchivo. cpe?Archivo=HKyt70eS6AGvSpdetlVqXsIX2kvla To-prEqTB-tPRc.

[16] Olonade, K., Jaji, M. \&Adekitan, O.: "Experimental comparison of selected pozzolanic materials". African Journal of Science, Technology, Innovation 
and Development, Vol. 9, № 4, (2017) 381-385.

[17] Akinyele, J. \&Ajede, A.: "The use of granulated plastic waste in structural concrete". African Journal of Science, Technology, Innovation and Development, Vol. 10, № 2, (2018) 169-175.

[18] Deo, S.: "Mix design approach for high 28 days' strength, high-volume, low-lime fly ash concrete".Road Materials and Pavement Design, Vol. 16, № 3, (2015) 707-715. DOI: $10.1080 / 14680629.2015 .1026381$

[19] Sociedad Americana para Pruebas y Materiales (ASTM): "Annual Book of ASTM Standards"., West Conshohocken, (2015).

[20] Servicio Ecuatoriano de Normalizacion, Norma tecnica de ecuatoriana, (NTE INEN 0157): "Cemento hidráulico. Determinación de la consistencia normal". Método de Vicat. Ecuador, (2009)

[21] Servicio Ecuatoriano de Normalizacion, Norma tecnica de ecuatoriana, (NTE INEN 0158): "Cemento hidráulico. Determinación del tiempo de fraguado". Método de Vicat, Ecuador, (2009).

[22] Servicio Ecuatoriano de Normalizacion, Norma tecnica ecuatoriana, (NTE INEN 0488): “Cemento hidráulico. Determinación de la resistencia a la compresión de morteros en cubos de $50 \mathrm{~mm}$ de arista". Ecuador, (2009).

[23] Servicio Ecuatoriano de Normalizacion, Norma tecnica ecuatoriana, (NTE INEN 0490): “Cementos hidráulicos compuestos”. Ecuador, (2011).

[24] Servicio Ecuatoriano de Normalizacion, Norma tecnica ecuatoriana, (NTE INEN 0855): “Áridos. Determinación de impurezas orgánicas en el árido fino para concreto". Ecuador, (2010).

[25] Servicio Ecuatoriano de Normalizacion, Norma tecnica ecuatoriana, (NTE INEN 0856): "Áridos. Determinación de la densidad, densidad relativa (gravedad específica) y absorción del árido fino". Ecuador, (2010).

[26] Servicio Ecuatoriano de Normalizacion, Norma tecnica ecuatoriana, (NTE INEN 0857): "Áridos. Determinación de la densidad, densidad relativa (gravedad específica) y absorción del árido grueso. Ecuador, (2010).
[27] Servicio Ecuatoriano de Normalizacion, Norma tecnica ecuatoriana, (NTE INEN 0858): "Áridos. Determinación de la masa unitaria (peso volumétrico) y el porcentaje de vacíos". Ecuador, (2010).

[28] Servicio Ecuatoriano de Normalizacion, Norma tecnica ecuatoriana, (NTE INEN 0860): "Aridos. Determinación del valor de la degradación del árido grueso de partículas menores a $37,5 \mathrm{~mm}$ mediante el uso de la máquina de los Ángeles". Ecuador, (2011).

[29] Servicio Ecuatoriano de Normalización, Norma técnica ecuatoriana, (NTE INEN 0638): "Bloques huecos de hormigón, definiciones, clasificaciones y condiciones generales". Ecuador, (1993).

[30] Servicio Ecuatoriano de Normalizacion, Norma tecnica ecuatoriana, (NTE INEN 0862): “Áridos para concreto. Determinación del contenido total de humedad". Ecuador, (2011).

[31] Ruma, F. y Well, A.: "Muchas caras del coeficiente de correlación". Journal of Statistics Education, Vol. 5, No. 3 (1997), 1-13.

[32] Harel, 0.: "La estimación de R2 y ajustada R2 en conjuntos de datos incompletos utilizando imputación múltiple". Journal of Applied Statistics, Vol. 36, No. 10 (2009), 1109-1118.

[33] Mohammad, K., Nopiah, Z.M., Ahmad Basri, N.E., Basri, H., Abushammala, M.F.M., and Maulud, K.N.A. "Previsión de residuos sólidos mediante la modelización ANFIS modificada". Journal of the Air \& Waste Management Association, Vol. 65, No. 10 (2015), 1229-1238.

[34] Geethu, V. y Santhoshkumar, S.: "Una eficiente realización de FPGA de detección de convulsiones a partir de la señal de EEG usando la transformación de ondas y características estadísticas". IETE Journal of Research, Vol. 66, No. 3 (2020), 315-325.

[35] Edmund, T., Jun, C., Hejazi, F. y Jaafar, M.: "Los desechos de plástico como reemplazo parcial de los agregados- son vista". IOP Conf. Series: Earth and Environmental Science, Vol. 357, No. 012018 (2019), 1-7. 


\section{REVISTA TECNICA}

DE LA FACULTAD DE INGENIERIA

UNIVERSIDAD DEL ZULIA

Vol. 44. N`1, Enero - Abril 2021, pp. 04 - 58

Esta revista fue editada en formato digital y publicada en Diciembre de 2020, por el Fondo Editorial Serbiluz, Universidad del Zulia. Maracaibo-Venezuela

www.luz.edu.ve

www.serbi.luz.edu.ve

www.produccioncientificaluz.org 